\title{
BACTERIAL CAUSES OF DROP IN EGG PRODUCTION IN LAYING HENS AND PREVENTION BY VACCINATION
}

\author{
Abd-elaleem Ismail ${ }^{1}$ and Ibrahem, $\mathrm{H}^{2}$ \\ ${ }^{1}$ Educational Hospital, Faculty of Vet. Med., Zagazig University. \\ ${ }^{2}$ Animal Health Research Institute, Serology Dept., Dokki, Giza
}

\begin{abstract}
Twenty-five laying flocks suffered from drop in egg production ranging from $3-10 \%$ were examined bacteriologically and the following bacteria were isolated: E.coli (21.9\%), Staphylococcus aureus (17.2\%) Pseudomonas aeruginosa(11.3\%), Proteus vulgaris (6.74\%), Enterococci (5.61\%), klebsiellaoxytoca (5.41\%), proteus mirabilis (5.41\%),klebsiella pneumoniae (5\%); klebsiellaozaenae (5\%), Yersiniaenterocolitica (4.18\%), Salmonella (4.08\%), Streptococcus (3.77\%), Actinomycesbiogenes(3.06\%), and Citrobacterfreundii (1.22\%). Two hundred and fifteen (215) E.coli isolates were obtained and serotyped as 0166 (45 isolate), 018 (31 isolate), 078 (29 isolate), 01 (28 isolate), 086(17 isolate), o20 (14 isolate) and untypedE.coli strains (13 isolate). The pathogenicity of these serotypes were determined in 9 days old chicks, the E.coli 0166infected chicks exhibited the higher morbidity and mortality $(42.9 \%$ 42.9\%) followed by E.coli 078, E.coli 0146,E.coli 020, E.coli 01 and E.coli 086 respectively. Experimental infection of laying hens with E.coli 0166 resulted in a significant decrease in egg production from the first week post infection up to the fifth week (13.5 - 35\%) followed by 018 and 078. vaccinated hens showed higher egg production, significant reduction in faecal shedding and egg contamination.
\end{abstract}




\section{INTRODUCTION}

Escherichia coli remains one of the most important pathogens in poultry, causing respiratory infection, cellulites, septicemia, or other diverse clinical condition (Barnes and Gross, 1997). E. coli is present in litter and dust increasing the risk of infection and making colibacillosis difficult to control. In addition, chickens do not naturally develop immunity to pathogenic serotypes. Using antibiotics to control E. coli infection results in transient, unreliable protection and often results in subsequent development of drug resistant E. coli strains (cloud et al.,1985 and DuPont and Steele, 1987).

Thus, vaccination could be important in controlling E. coli., althoughmonovalent E. coli vaccines did not protect against other E. coli serotypes.

Therefore, an effective vaccine against colibacillosis should contain the serotypes of $E$. coli commonly isolated from local farms and associated with this infection (Gyimahet al.,1985).

Mahmood and Reza, (2010) isolated E. coli from oviduct of layer hens $(30-68 \mathrm{w}$.$) , isolates belonged to 11$ different serogroups including $01,02,08,015,020,025,036,078,086$ and 0111 . 078 was the most prevalent serotype followed by 02 and 01 . Infection of 1 day old chicks via intraperitoneal or oral route with E. coli 0128 produced $(90 \%-70 \%)$ mortality respectively, for 078 (90-50\%), for $0166(80 \%$ - 60\%), for 029 (80\%-50\%) and for $01(70 \%-60 \%)$ respectively, (Ahmed, 2012). 
Hegazyet al., (2012) vaccinated two groups of breeder hens with $E$. coli sonicated or formalized vaccine twice at 20 and 25 week of age followed by Evaluation of antibodies by Elisa and indirect haemagglutination test from hens, eggs and hatched chicks. Sonicated vaccine produced significantly higher titer, protection and body weight than formalinized vaccine. Immunization of breeders protects offspring with maternal antibodies from E. coli infection up to the fourth week of age. The aim of this study is the detection of E. coli causing decreased egg production and prevention by vaccination.

\section{MATERIAL AND METHODS}

\section{1- Samples:}

One hundered and fifty cloacal swabs from apparently healthy layer chickens. Lung, liver, intestine, ovary and oviduct from 100 diseased layer chickens and 100 freshly dead laying chickens were collected from different farms which suffered from a decrease in egg production and / or high mortalities.

1- Bacterial isolation from collected samples according to Buchanan and Gibbons (1974).

2- Bacteriological identification, according to Cruickshank,et al.,(1975).

3- Serotyping, according to Edwards and Ewings (1972).

4- Streptomycin resistant challenge strains field isolates of E. coli 078, 0166, 01 and 018, were rendered streptomycin resistant before experimental use according to Barnhart et al.,(1999). 
5- Bacterial titration: according to Sambrooket al.,(1989).

6- Preparation of whole cell, formalin inactivated polyvalent $E$. colibacterin, according to the method of Panigraphyet al., (1983).

7- Bacteriological examination of eggs: three eggs per group were examined along 5 weeks post infection.

8- Cloacal swabs: six swabs per group were collected along 5 weeks post infection.

\section{2- Birds:}

a- Fifty six, 9 days old chicks housed on floor and received prepared ration $(21 \%$ protein, $5.14 \%$ fat, $2950 \mathrm{kc} / \mathrm{kg})$.

b- Sixty 19 weeks old laying hens (Bovans) housed on flour and received a prepared ration $18 \%$ protein, $5.8 \%$ fat and energy 2780 $\mathrm{kc} / \mathrm{kg}$.

\section{3- Experimental design:}

Experiment (1) study the pathogenicity of the isolated E.coli to 9 day oldchicken, table (1).

Experiment (2) aimed to study the effect of isolated E.coli strains on egg production, faecal shedding and egg contamination in laying hens and prevention by vaccination table (2).

Statistical analysis: Data were analyzed using SAS statistical analysis system package (SAS, 2002). One way ANOVA was performed to determine the differences among groups. 
Bacterial Causes Of Drop In Egg Production In ...

Table (1): pathagenicity of E.coli serotypes in 9 days old chicks.

\begin{tabular}{|c|c|c|c|c|}
\hline Group & Number & Infection & Route & Dose \\
\hline $\begin{array}{l}1 \\
2 \\
3 \\
4 \\
5 \\
6 \\
7 \\
\end{array}$ & $\begin{array}{l}7 \\
7 \\
7 \\
7 \\
7 \\
7 \\
7 \\
\end{array}$ & $\begin{array}{c}\text { E.coli O78 } \\
\text { E.coli O } 18 \\
\text { E.coli O166 } \\
\text { E.coli O146 } \\
\text { E.coli O20 } \\
\text { E.coli O1 } \\
\text { E.coli O86 } \\
\end{array}$ & \multirow[t]{2}{*}{ 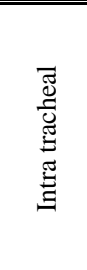 } & \multirow[t]{2}{*}{ 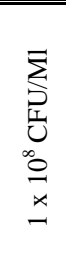 } \\
\hline 8 & $\overline{77}$ & Non infected & & \\
\hline
\end{tabular}

Table (2): Efficacy of vaccination of laying hens with prepared polyvalent $E$. Colibacterin (078, 0166, 01 and 0146) in prevention of E. coli infection $(n=6)$

\begin{tabular}{|c|c|c|c|c|c|c|c|c|c|}
\hline \multirow{2}{*}{ Group } & \multirow{2}{*}{ No. } & \multicolumn{4}{|c|}{ Vaccination } & \multicolumn{4}{|c|}{ E. Coli } \\
\hline & & Type & Age & Dose & Route & Type & Age & Dose & Route \\
\hline Vacc .ge & 1 & $\begin{array}{c}\text { Poly valent } \\
\text { inactivated E.coli } \\
\text { vaccine }\end{array}$ & $\begin{array}{c}\text { At } 19 \text { and } 21 \mathrm{w} \text { of } \\
\text { age }\end{array}$ & $0.5 \mathrm{ml}$ & $\mathrm{S} / \mathrm{C}$ & $\mathrm{O} 78$ & $23 \mathrm{w}$ & \multirow{10}{*}{ 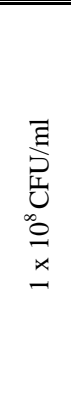 } & \multirow{10}{*}{ 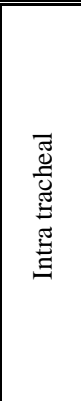 } \\
\hline & 2 & & & & & O 166 & & & \\
\hline & 3 & & & & & $\mathrm{O} 1$ & & & \\
\hline & 4 & & & & & O 18 & & & \\
\hline & 5 & & & & & NI & & & \\
\hline Positive control & 6 & NV & & & & $\mathrm{O} 78$ & & & \\
\hline & 7 & & & & & O166 & & & \\
\hline & 8 & & & & & O01 & & & \\
\hline & 9 & & & & & O18 & & & \\
\hline negative control & 10 & $\mathrm{NV}$ & & & & NI & & & \\
\hline
\end{tabular}

\section{RESULTS}

\section{Isolation of bacteria:}

The incidence of the isolated bacteria was E.coli (21.9\%), Staphylococcusaurus (17.2\%), Pseudomonas aeruginosa(11.3\%), Proteus vulgaris (6.74\%),Enterococci (5.61\%), Klebsiellaoxytoca (5.41\%), Proteus mirabilis (5.41\%), klebsiellapneumoniae(5\%), Klebsiellaozaenae(5\%), Yersinia enterocolitica (4.18\%), Salmonella (4.08\%), Streptococcus species (3.77\%), Actimomycesbiogenes (3.06\%) 
and Citrobacterfreundii (1.22\%). Two hundered and fifteen (215) E.coli isolates were serotyped as 0166 (45 isolate), 0146 (38 isolate), 018(31 isolate), 078(29 isolate), 01 (28 isolate), 086 (17 isolate), 020 (14 isolate) and untypedE.coli strains (13 isolate)

\section{Pathogenicity of E.Coli strains to 9 daysold chicks:}

E.coli 0166 was more pathogenic for 9 days old chicks, and resulted in higher mortality and morbidity $(42.9 \%$ and $42.9 \%$ respectively) followed by 018 ( $28.6 \%$ and 28.6 respectively). 01 ( $28.6 \%$ and $14.3 \%$ respectively), 0146 ( $14.3 \%$ and $28.6 \%$ respectively), 078 (zero $\%$ and $42.9 \%$ respectively), 020 (zero\% and $14.3 \%$ respectively) and 086 ( zero\% and $14.3 \%$ respectively).

\section{Effect of E.coli serotypes on Egg production:}

E.coli 0166 infection to laying hens induced significant decrease in egg production starting from the $1^{\text {st }}$ week post infection up to the $5^{\text {th }}$ w.P.i. (16.67\%- 35.77\%) followed by E.coli 01 the drop was from the $2^{\text {nd }}$ W.P.I. $(11.43 \%-28.57 \%)$

Effect of vaccination with E.Colibacterin on egg production, faecal shedding post challenge, and egg contamination:

No significant increase post vaccination and challenge by 078, 01, 018, while significant increase in egg production was observed post challenge with 0166 (group, 2) from the $1^{\text {st }}$ W.P.I. up to the $5^{\text {th }}$ W.P.I. $(100 \%)$ in comparison with non vaccinated infected group (7) with egg production (64.23\%) table(3). Faecal shedding decreased (078) post vaccination from the $1^{\text {st }}$ WPI from $53.3 \%$ to $1.11 \%$ in the non vaccinated infected and vaccinated group respectively and reached to zero shedding at the $5^{\text {th }}$ WPI in the vaccinated birdscompaired to $30 \%$ in infected non 
vaccinated birds, while the bacterin decreased E.coli 0166 shedding from $27.8 \%$ to $5.55 \%$ in non vaccinated infected and vaccinated groups respectively and reached to zero at the end of the experiment in vaccinated birds. (Table, 4).The E.colibacterin decrease the egg albumen contamination with E.coli 078,0166 from $44.4 \%$ and $33.3 \%$ to zero respectively.

Table (3): Effect of tetravalent bacterin, $(078,0166,01,0146)$ on egg production in E.coli- infected Bovans hens

\begin{tabular}{|c|c|c|c|c|c|c|c|c|}
\hline \multirow{2}{*}{ Groups } & \multirow{2}{*}{$\begin{array}{l}\text { No of } \\
\text { birds }\end{array}$} & \multirow{2}{*}{$\begin{array}{l}\text { Vaccin- } \\
\text { ation }\end{array}$} & \multirow{2}{*}{ Infection } & \multicolumn{5}{|c|}{ Egg production \% weeks PI } \\
\hline & & & & $1^{\text {st }}$ week & $2^{\text {nd }}$ week & $3^{\text {rd }}$ week & $4^{\text {th }}$ week & $5^{\text {th }}$ week \\
\hline 1 & 6 & $+\mathrm{ve}$ & E.coli 078 & $92.86^{\mathrm{ab}} \pm 3.37$ & $92.86^{\mathrm{ab}} \pm 3.37$ & $92.86^{\mathrm{ab}} \pm 3.37$ & $92.86^{\mathrm{ab}} \pm 3.37$ & $95.24^{\mathrm{a} \pm 3.07}$ \\
\hline 2 & 6 & $+\mathrm{ve}$ & E.coli 0166 & $100^{\mathrm{a}} \pm 0.0$ & $97.62^{\mathrm{ab}} \pm 2.3 .8$ & $97.62^{\mathrm{a}} \pm 2.38$ & $100^{\mathrm{a}} \pm 0.0$ & $90.47^{\mathrm{a}} \pm 4.96$ \\
\hline 3 & 6 & $+\mathrm{ve}$ & E.coli01 & $90.47^{\mathrm{b}} \pm 4.96$ & $90.47^{\mathrm{ab}} \pm 4.96$ & $92.86^{\mathrm{a}} \pm 3.37$ & $92.86^{\mathrm{ab}} \pm 3.37$ & $92.86^{\mathrm{a}} \pm 3.37$ \\
\hline 4 & 6 & $+\mathrm{ve}$ & E.coli 018 & $97.62^{\mathrm{ab}} \pm 2.38$ & $95.24^{\mathrm{ab}} \pm 3.07$ & $92.86 \mathrm{a} \pm 4.96$ & $90.47^{\mathrm{ab}} \pm 3.37$ & $90.47^{\mathrm{a}} \pm 4.95$ \\
\hline 5 & 6 & $+v e$ & $\begin{array}{c}\text { Non } \\
\text { infected }\end{array}$ & $97.62^{\mathrm{ab}} \pm 2.38$ & $92.86^{\mathrm{ab} \pm 3.37}$ & $97.62^{\mathrm{a}} \pm 2.38$ & $97.61^{\mathrm{ab}} \pm 2.38$ & $95.23^{\mathrm{a}} \pm 3.07$ \\
\hline 6 & 5 & -ve & E.coli078 & $100^{\mathrm{a}} \pm 0.0$ & $100^{\mathrm{a}} \pm 0.0$ & $91.43^{\mathrm{a}} \pm 4.04$ & $91.43^{\mathrm{ab}} \pm 4.04$ & $91.43^{\mathrm{a}} \pm 4.04$ \\
\hline 7 & 6 & -ve & E.coli 0166 & $83.33^{\mathrm{c}} \pm 1.01$ & $73.80^{\circ} \pm 3.37$ & $66.66^{c} \pm 1.57$ & $64.23^{\mathrm{c}} \pm 2.38$ & $64.28^{\mathrm{c}} \pm 2.38$ \\
\hline 8 & 5 & -ve & E.coli01 & $97.14^{\mathrm{ab}} \pm 286$ & $88.57^{\mathrm{b}} \pm 4.04$ & $80.00^{\mathrm{b}} \pm 0.0$ & $71.43^{c} \pm 5.95$ & $74.29^{\mathrm{bc}} \pm 5.71$ \\
\hline 9 & 5 & $-v e$ & E.coli018 & $100^{\mathrm{a}} \pm 0.0$ & $94.29^{\mathrm{ab}} \pm 3.69$ & $91.43^{\mathrm{a}} \pm 4.04$ & $85.71^{\mathrm{b}} \pm 3.69$ & $88.57^{\mathrm{a}} \pm 4.04$ \\
\hline 10 & 6 & -ve & $\begin{array}{c}\text { Non } \\
\text { infected }\end{array}$ & $97.62^{\mathrm{ab}} \pm 2.38$ & $100^{\mathrm{a}} \pm 0.0$ & $95.24^{\mathrm{a}} \pm 3.07$ & $100^{\mathrm{a}} \pm 0.0$ & $97.38^{\mathrm{a}} \pm 2.38$ \\
\hline
\end{tabular}

* All birds of groups $(1,2,3,4,5)$ were vaccinated subcutaneously with $0.5 \mathrm{ml}$ of polyvalent E.colibacterin (078, 0166, 01 and 0146) twice at 19 and 21 weeks of age

**All birds groups $(1,2,3,46,7,8,9)$ were infected intratrachealy with $1 \times 108 \mathrm{CFU} / \mathrm{ml}$ of different E.coli serotypes at 23 weeks of age

***Values within the same column bearing different superscripts are significant at $p \leq 0.05$ 
Abd-elaleem Ismaill \& Ibrahem, H..

Table (4): Effect of tetravalent E.colibacterin $(0.78,0166,01$ and 0146) on E.colifaecal shedding in bovans layers.

\begin{tabular}{|c|c|c|c|c|c|c|c|c|c|c|c|c|c|c|c|}
\hline \multirow{3}{*}{ Groups } & \multirow{3}{*}{$\begin{array}{c}\text { No } \\
\text { of } \\
\text { birds }\end{array}$} & \multirow{3}{*}{$\begin{array}{l}\text { Vaccin } \\
\text {-ation }\end{array}$} & \multirow{3}{*}{ Infection } & \multicolumn{12}{|c|}{ E.colifaecal shedding } \\
\hline & & & & \multicolumn{2}{|c|}{$1^{\text {st }}$ week } & \multicolumn{2}{|c|}{$2^{\text {nd }}$ week } & \multicolumn{2}{|c|}{$3^{\text {rd }}$ week } & \multicolumn{2}{|c|}{$4^{\text {th }}$ week } & \multicolumn{2}{|c|}{$5^{\text {th }}$ week } & \multicolumn{2}{|c|}{ Total } \\
\hline & & & & No. & $\%$ & No. & $\%$ & No. & $\%$ & No. & $\%$ & No. & $\%$ & No. & $\%$ \\
\hline 1 & 6 & $+\mathrm{ve}$ & E.coli 078 & $2 / 18$ & 1.11 & $2 / 12$ & 16.66 & $0 / 12$ & 0.0 & $1 / 12$ & 8.33 & $0 / 12$ & 0.0 & $5 / 66$ & $7.5^{\mathrm{c}}$ \\
\hline 2 & 6 & $+\mathrm{ve}$ & E.coli0166 & $1 / 18$ & 5.55 & $0 / 12$ & 0.00 & $1 / 12$ & 8.33 & $0 / 12$ & 0.0 & $0 / 12$ & 0.0 & $2 / 66$ & $30.03^{\mathrm{d}}$ \\
\hline 3 & 6 & $+\mathrm{ve}$ & E.coli01 & $6 / 17$ & 35.29 & $2 / 12$ & 16.66 & $1 / 12$ & 8.33 & $2 / 12$ & 16.66 & $2 / 12$ & 16.66 & $13 / 65$ & $20^{\mathrm{bc}}$ \\
\hline 4 & 6 & $+\mathrm{ve}$ & E.coli018 & $5 / 18$ & 27.77 & $2 / 12$ & 16.66 & $0 / 12$ & 0.0 & $3 / 12$ & 25.0 & $3 / 12$ & 25.0 & $13 / 66$ & $19.7^{\mathrm{bc}}$ \\
\hline 5 & 6 & $+\mathrm{ve}$ & Non infected & $0 / 18$ & 0.0 & $0 / 12$ & 0.0 & $0 / 12$ & 0.0 & $0 / 12$ & 0.0 & $0 / 12$ & 0.0 & $0 / 66$ & $0.0^{\mathrm{d}}$ \\
\hline 6 & 6 & $-\mathrm{ve}$ & $\begin{array}{l}\text { E.coli078 } \\
\end{array}$ & $8 / 15$ & 53.33 & $4 / 10$ & 40.0 & $2 / 10$ & 20.0 & $5 / 10$ & 50.0 & $3 / 10$ & 30.0 & $22 / 55$ & $40.0^{\mathrm{a}}$ \\
\hline 7 & 6 & -ve & E.coli0166 & $5 / 18$ & 27.77 & $2 / 12$ & 16.66 & $3 / 12$ & 25.0 & $1 / 12$ & 8.33 & $1 / 12$ & 8.33 & $13 / 66$ & $19.7^{\mathrm{bc}}$ \\
\hline 8 & 6 & -ve & E.coli01 & $5 / 15$ & 33.33 & $4 / 10$ & 40.0 & $1 / 10$ & 10.0 & $3 / 10$ & 30.0 & $2 / 10$ & 20.0 & $15 / 55$ & $25.9^{\mathrm{b}}$ \\
\hline 9 & & -ve & E.coli 018 & $8 / 15$ & 53.33 & $4 / 10$ & 40.0 & $4 / 10$ & 40.0 & $4 / 10$ & 40.0 & $3 / 10$ & 30.0 & $23 / 55$ & $39.7^{\mathrm{a}}$ \\
\hline 10 & 6 & $\begin{array}{c}\text { Non } \\
\text { vaccinat } \\
\text { ed }\end{array}$ & Non infected & $0 / 18$ & 0.0 & $0 / 12$ & 0.00 & $0 / 12$ & 0.0 & $0 / 12$ & 0.0 & $0 / 12$ & 0.0 & $0 / 66$ & $0.0^{\mathrm{d}}$ \\
\hline
\end{tabular}

* All birds of groups $(1,2,3,4,5)$ were vaccinated subcutaneously with $0.5 \mathrm{ml}$ of polyvalent E.colibacterin (078, 0166,01 and 0146) twice at 19 and 21 weeks of age

** All birds groups $(1,2,3,46,7,8,9)$ were infected intratrachealy with $1 \mathrm{x} 108 \mathrm{CFU} / \mathrm{ml}$ of different E.coli serotypes at 23 weeks of age

*** Values within the same column bearing different superscripts are significant at $\mathrm{p} \leq 0.05$

Table (5): Effect of tetravalent E.colibacterin (0.78, 0166, 01 and 0146) on reisolation of E.coli serotypes from egg albumen in bovans laying hens.

\begin{tabular}{|c|c|c|c|c|c|c|c|c|c|c|c|c|c|c|c|}
\hline \multirow{3}{*}{ Groups } & \multirow{3}{*}{$\begin{array}{c}\text { No } \\
\text { of } \\
\text { birds }\end{array}$} & \multirow{3}{*}{$\begin{array}{l}\text { Vaccin } \\
\text {-ation }\end{array}$} & \multirow{3}{*}{ Infection } & \multicolumn{12}{|c|}{ E.coliresisolation } \\
\hline & & & & \multicolumn{2}{|c|}{$1^{\text {st }}$ week } & \multicolumn{2}{|c|}{$2^{\text {nd }}$ week } & \multicolumn{2}{|c|}{$3^{\text {rd }}$ week } & \multicolumn{2}{|c|}{$4^{\text {th }}$ week } & \multicolumn{2}{|c|}{$5^{\text {th }}$ week } & \multicolumn{2}{|c|}{ Total } \\
\hline & & & & No. & $\%$ & No. & $\%$ & No. & $\%$ & No. & $\%$ & No. & $\%$ & No. & $\%$ \\
\hline 1 & 6 & $+\mathrm{ve}$ & E.coli 078 & $0 / 9$ & 0.0 & $0 / 6$ & 0.0 & $0 / 6$ & 0.0 & $1 / 9$ & 11.11 & $0 / 6$ & 0.0 & $1 / 36$ & $2.77^{b}$ \\
\hline 2 & 6 & $+\mathrm{ve}$ & E.coli0166 & $1 / 9$ & 11.11 & $0 / 6$ & 0.0 & $0 / 6$ & 0.0 & $0 / 9$ & 0.0 & $1 / 6$ & 16.66 & $2 / 36$ & $5.55^{\mathrm{b}}$ \\
\hline 3 & 6 & $+\mathrm{ve}$ & E.coli01 & $1 / 9$ & 11.11 & $1 / 6$ & 16.66 & $0 / 6$ & 0.0 & $0 / 9$ & 0.0 & $1 / 6$ & 16.66 & $3 / 36$ & $8.33^{\text {ab }}$ \\
\hline 4 & 6 & $+\mathrm{ve}$ & E.coli018 & $3 / 9$ & 33.33 & $1 / 6$ & 16.66 & $0 / 6$ & 0.0 & $1 / 9$ & 11.11 & $1 / 6$ & 16.66 & $6 / 36$ & $16.7^{\text {ab }}$ \\
\hline 5 & 6 & $+\mathrm{ve}$ & Non infected & $0 / 9$ & 0.0 & $0 / 6$ & 0.0 & $0 / 6$ & 0.0 & $0 / 9$ & 0.0 & $0 / 6$ & 0.0 & $0 / 36$ & $0.0^{\mathrm{b}}$ \\
\hline 6 & 5 & $-\mathrm{ve}$ & \begin{tabular}{|l|} 
E.coli078 \\
\end{tabular} & $4 / 9$ & 44.44 & $0 / 6$ & 0.0 & $0 / 6$ & 0.0 & $0 / 9$ & 0.0 & $0 / 6$ & 0.0 & $4 / 36$ & $11.1^{\mathrm{ab}}$ \\
\hline 7 & 6 & $-\mathrm{ve}$ & E.coli0166 & $0 / 9$ & 0.0 & $2 / 6$ & 33.33 & $1 / 6$ & 16.66 & $2 / 9$ & 22.22 & $0 / 6$ & 0.0 & $5 / 36$ & $13.9^{\mathrm{ab}}$ \\
\hline 8 & 5 & $-\mathrm{ve}$ & E.coli 01 & $1 / 6$ & 16.66 & $2 / 6$ & 33.33 & $0 / 6$ & 0.0 & 09 & 0.0 & $1 / 6$ & 16.66 & $4 / 33$ & $12.1^{\mathrm{ab}}$ \\
\hline 9 & 5 & -ve & E.coli018 & $4 / 6$ & 66.66 & $2 / 6$ & 33.33 & $0 / 6$ & 0.0 & $2 / 9$ & 22.22 & $1 / 6$ & 16.66 & 9/33 & $27.3^{\mathrm{a}}$ \\
\hline 10 & 6 & \begin{tabular}{|c|} 
Non \\
vaccinat \\
ed
\end{tabular} & Non infected & $0 / 9$ & 0.0 & $0 / 6$ & 0.0 & $0 / 6$ & 0.0 & $0 / 9$ & 0.0 & $0 / 6$ & 0.0 & $0 / 36$ & $0.0^{\mathrm{b}}$ \\
\hline
\end{tabular}

*All birds of groups $(1,2,3,4,5)$ were vaccinated subcutaneously with $0.5 \mathrm{ml}$ of polyvalent E.colibacterin $(078,0166,01$ and 0146) twice at 19 and 21 weeks of age

**All birds groups $(1,2,3,4,6,7,8,9)$ were infected intratrachealy with 1 x $108 \mathrm{CFU} / \mathrm{ml}$ of different E.coli serotypes at 23 weeks of age

$* * *$ Values within the same column bearing different superscripts are significant at $\mathrm{p} \leq 0.05$

$\overline{\text { Kafrelsheikh Vet. Med. J. Vol. } 15 \text { No. } 1 \text { (2017) }}$ 
Table (6): Effect of tetravalent E.colibacterin $(0.78,0166,01$ and 0146) on reisolation of E.coli serotypes from egg yolk In Bovanslayers.

\begin{tabular}{|c|c|c|c|c|c|c|c|c|c|c|c|c|c|c|c|}
\hline \multirow[t]{3}{*}{ Groups } & \multirow{3}{*}{\begin{tabular}{|c} 
No \\
of \\
birds
\end{tabular}} & \multirow{3}{*}{$\begin{array}{l}\text { Vaccin } \\
\text {-ation }\end{array}$} & \multirow{3}{*}{ Infection } & \multicolumn{12}{|c|}{ E.coli faecal shedding } \\
\hline & & & & \multicolumn{2}{|c|}{$1^{\text {st }}$ week } & \multicolumn{2}{|c|}{$2^{\text {nd }}$ week } & \multicolumn{2}{|c|}{$3^{\text {rd }}$ week } & \multicolumn{2}{|c|}{$4^{\text {th }}$ week } & \multicolumn{2}{|c|}{$5^{\text {th }}$ week } & \multicolumn{2}{|c|}{ Total } \\
\hline & & & & No. & $\%$ & No. & $\%$ & No. & $\%$ & No. & $\%$ & No. & $\%$ & No. & $\%$ \\
\hline 1 & 6 & $+\mathrm{ve}$ & E.coli 078 & $0 / 9$ & 0.0 & $0 / 6$ & 0.0 & $0 / 6$ & 0.0 & $0 / 9$ & 0.0 & $0 / 6$ & 0.0 & $0 / 36$ & $0.0^{\mathrm{c}}$ \\
\hline 2 & 6 & $+\mathrm{ve}$ & E.coli0166 & $1 / 9$ & 11.11 & $1 / 6$ & 16.66 & $0 / 6$ & 0.0 & $0 / 9$ & 0.0 & $0 / 6$ & 0.0 & $2 / 36$ & $.55^{\mathrm{bc}}$ \\
\hline 3 & 6 & $+\mathrm{ve}$ & E.coli01 & $0 / 9$ & 0.0 & $1 / 6$ & 16.66 & $1 / 6$ & 16.66 & $1 / 9$ & 11.11 & $1 / 6$ & 16.66 & $4 / 36$ & $11.1^{\mathrm{abc}}$ \\
\hline 4 & 6 & $+\mathrm{ve}$ & E.coli018 & $3 / 9$ & 33.33 & $1 / 6$ & 16.66 & $0 / 6$ & 0.0 & $0 / 9$ & 0.0 & $0 / 6$ & 0.0 & $4 / 36$ & $11.1^{\mathrm{abc}}$ \\
\hline 5 & 6 & $+\mathrm{ve}$ & Non infected & $0 / 9$ & 0.0 & $0 / 6$ & 0.0 & $0 / 6$ & 0.0 & $0 / 9$ & 0.0 & $0 / 6$ & 0.0 & $0 / 36$ & $0.0^{\mathrm{c}}$ \\
\hline 6 & 5 & -ve & E.coli078 & $1 / 9$ & 11.11 & $0 / 6$ & 0.0 & $1 / 6$ & 16.66 & $1 / 9$ & 11.11 & $0 / 6$ & 0.0 & $3 / 36$ & $8.33^{\mathrm{abc}}$ \\
\hline 7 & 6 & $-\mathrm{ve}$ & E.coli0166 & $2 / 9$ & 22.22 & $3 / 6$ & 50.0 & $0 / 6$ & 0.0 & $0 / 9$ & 0.0 & $1 / 6$ & 16.66 & $6 / 36$ & $16.7^{\mathrm{ab}}$ \\
\hline 8 & 5 & -ve & E.coli01 & $2 / 6$ & 33.33 & $1 / 6$ & 16.66 & $2 / 6$ & 33.33 & $1 / 9$ & 11.11 & $1 / 6$ & 16.66 & $7 / 33$ & $21.2^{\mathrm{a}}$ \\
\hline 9 & 5 & -ve & E.coli018 & $0 / 6$ & 0.0 & $2 / 6$ & 33.33 & $2 / 6$ & 33.33 & $2 / 9$ & 22.22 & $0 / 6$ & 0.0 & $6 / 33$ & $18.2^{\mathrm{ab}}$ \\
\hline 10 & 6 & $\begin{array}{c}\text { Non } \\
\text { vaccinat } \\
\text { ed }\end{array}$ & Non infected & $0 / 9$ & 0.0 & $0 / 6$ & 0.0 & $0 / 6$ & 0.0 & $0 / 9$ & 0.0 & $0 / 6$ & 0.0 & $0 / 36$ & $0.0^{\mathrm{c}}$ \\
\hline
\end{tabular}

*All birds of groups $(1,2,3,4,5)$ were vaccinated subcutaneously with $0.5 \mathrm{ml}$ of polyvalent E.colibacterin $(078,0166,01$ and 0146) twice at 19 and 21 weeks of age

**All birds groups $(1,2,3,46,7,8,9)$ were infected intratrachealy with 1 x $108 \mathrm{CFU} / \mathrm{ml}$ of different E.coli serotypes at 23 weeks of age

***Values within the same column bearing different superscripts are significant at $\mathrm{p} \leq 0.05$

\section{DISCUSSION}

Bacterial isolation from cloacal swabs and tissuesfrom laying hens resulted in E.coli (21.9\%),Staphylococcus aureus(17.2\%),Pseudomonas aeruginosa(11.3\%),Proteus vulgaris (6.74\%),Enterococci (5.61\%), klebsiellaoxytoca (5.41\%), Proteus mirabilis(5.41\%), Klebsiellapnemonae (5\%), klebsiellaozaenae (5\%), Yersinia enterocolitica (4.18\%), Salmonella (4.08\%), Streptococcus species (3.77\%), Actinomycesbiogenes (3.06\%) and Citrobacter freundii (1.22\%). Similarly Mubaraket al., (1998) isolated E.coli (8.1\%), Salmonella enteritidis (14.5\%), Proteus Vulgaris (4.8\%), S.typhyimurium (14.5\%), Proteus mirabilis (11.3\%), Klebsiellaoxytoca (20.9\%), Klebsiellapnemoniae (11.3\%), Citrbacter 
cloacae (6.5\%) and Yersinia enterocolitica (8.1\%) from ovary and oviduct of freshly dead laying hens. E.coli isolates were serotyped as E.coli O166 (20.9\%), O146 (17.7\%), O18 (14.4\%), O78 (13.5\%), O1 (13\%), O86 (7.9\%), and O20 (6.5\%), also several studies on laying hens suffered from mortalities and drop in egg production with positive isolation of E.coli from oviduct were recorded by Vandekerchoveet al., (2004), Zanellaet al., (2000), Mahmood and Reza (2010) and Oh et al.,(2011). Exprimentally E.coli $\mathrm{O} 166$ was more pathogenic for 9 days old chicks with high morbidity and mortality $(42.9 \%, 42.9 \%)$ respectively followed by $\mathrm{O} 18(28.6 \%, 28.6 \%), \mathrm{O} 1(14.3 \%, 28.6 \%)$, $\mathrm{O} 146(28.6 \%, 14.3 \%), \mathrm{O} 78(42.9 \%, 0 \%), \mathrm{O} 20(14.3 \%, 0 \%)$ and $\mathrm{O} 86$ $(14.3 \%, 0 \%)$ respectively.High mortality was recorded by Rosenberger et al., (1985) and Heller et al., (1990).

Vaccination of laying hens with polyvalent E.colibacterinfollowed by challenge with E.coli O166 two weeks p.v. induced significant increase in egg production througout the experiment period, while E.coli serotypes $\mathrm{O} 78$ and $\mathrm{O} 18$ showed non significant increase in egg production. Vaccination with polyvalent E.coli was effective in decreasing faecal shedding of E.coli serotypes O78, O166 and O18 as early as $1^{\text {st }}$ week post infection. The significant protection from E.coli $\mathrm{O} 166$ and $\mathrm{O} 1$,could be attributed to lowering colonization of E.coli due to IgA which protect mucosa from E.coli infection (Ograet al.,1994). Also vaccination with polyvalent E.colibacterin decreased egg albumen contamination, No E.coli $\mathrm{O} 78$ reisolation along the experiment except the $4^{\text {th }}$ week p.I. (1+ve from 9), O166,0\% from the $2^{\text {nd }}$ week up to the end, except the $1^{\text {st }}$ and $5^{\text {th }}$ w.(1 +ve only ), while $\mathrm{O} 1$ and $\mathrm{O} 18$ was still found in faeces and egg albumin which may be due to colonization of 
the oviduct with E.coli strains.Reisolation of E.coli strains from egg yolk post vaccination and infection revealed negative result for $\mathrm{O} 78$ along the experiment, while low rate ofreisolation of $\mathrm{O} 166$ and $\mathrm{O} 18$ was in the 1st two weeks p.i only and $0 \%$ up to the end $\mathrm{O} 1$ was still found but in lower frequency when compared with infected non vaccinated group. Similar protective effect was reported post vaccination by Gyimah and Panigraphy (1985), and Huang and Matsumoto (1998).

It was concluded that different E.coli serotypes infection in laying hens cause a variable decrease in the egg production,colonize the intestinal and reproductive tracts and contaminate eggs. Vaccination was efficient in controlling homologus and partially heterologous E.coli infection.It is clear that preparation of local vaccine from the most prevalent pathogenic E.coli serotypes circulating in the area was protective.

\section{REFERENCES}

- Ahmed, H.M.(2012): characterization of common antegens shared by $\mathrm{E}$.coli strains causing diseases in poultry .Ph.D thesis, dep. of microbiology faculty of vet medicine kafrek sheikh in Egypt.

- Barnes, H.J. and gross, W.B. (1997): colibacillosis in disease of poultry, $10^{\text {th }}$ ed. Jowa state university press, Ames.P.131-141

- Barnhart.E.,Caldwell ,D,croush , M.,Byra, J.,Corrier , D. and Hargis, B. (1999): Effect of lactose administration in the drinking water prior to and during feed withdrawal . poultrysci 78:211-214

- Buchanon, R.E. and Gibbons, N.E. (1974): Bergeyss manual of determinative bacteriology, $8^{\text {th }}$ ed Williams and wilkins .co, Blatimose. 
- Cloud, S.S., Rosenberges, J.K. , Fries, P.A., Wilson, R.A. and Odor , E.M. (1985): invitro and invivo characterization of avian E.coli serotypes, metabolic activity and antibiotic sensitivity . Avian Dis. 29:1084-1093.

- Cruickshank, K.R., Duguiel, J.P., Marmion, B.P. and swain, R.H.A. (1975): Medical microbiology . $12^{\text {th }}$ ED vol II Churchill .livingstone, Edenburg . London and New York.

- Dupont, H.L. and steele . J.H. (1987): use of antimicrobial agents in animal feeds, implications for human health. Rev. infect. Dis. $9: 447-460$

- Edwards, P. R. and Ewings, W. H. (1972): Identification of Enterobacteriacae. $3^{\text {rd }}$ Ed.,Burgess publishing Co. Unineopoles .

- Gyimah, J.E., Panigraphy, B.,Hall, C.F. and Williams, J. D. (1985): Immunogenicity of an-Emulsified E.colibacterin against heterologous challenge. Avian Dis. 29 (2) :540-545.

- Hegazy,A. M., Lebda, M. A., Abdelsamie, L. K., Abdallah, H.A. and Abdallah, E. M. (2010): Comparative study on sonicated and formalin inactivated E.coli vaccines in chickens. $7^{\text {th }}$ Int. Sci. Conf. Mansura. 501-510.

- Heller, E.D., Leitner, G., Drabakin, N. and Meamed,D. (1990): Passive immunization of chicks against E.coli . Avian Pathology. 19: 345-354.

- Huang,H. J. and Matsumoto, M.(1998): Immunity against E.coli infection in chickens assessed by viable bacterial counts in internal organs .Avian Dis. 43: 469-475. 
- Mahmood, S. and Reza, G.(2010): Characterization of E.coli isolates from commercial layer hens salpingitis. Amer.J. of animal and veterinary Sci.5(3): 208-214.

- Ogra, P.L., Strober, W., Mestecky, J., MeGhee, J. R., Lamm, M. E. and Bienenstock, J. (1994): Hand book of Mucosal immunology .Academic press, Inc., San. Diego. New York. Boston. London. Sydney. Tokyo. Toronto.

- Panigraphy, B.,Gyimah, J.E. , Hall, C.F. and Williams, J.D. (1983): Immunogenic potency of an oil emulsified E.coli bacteria . Avian Dis 28(2): 475-481.

- Rosenberges, J.K. , Fries, P.A. and Cloud, S.S. (1985): Invitro and invivo characterization of avian E.coli iii . Immunization Avian Dis. 29(4): 1108-1117.

- Sambrook, J., Fritsch, E.F. and Mawiatis , Y. (1989): Molecular cloning a laboratory

- Vandekerchove , D. , DeHerdt , P. , H. and Pasmans , F. (2004): colibacillosis in caged layer hens : characterization of disease and the etiological agent . Avian pathol . 33(2) :117-125.

- Zanella, A., Alborali, G.L., Basdotti, M., Candotti, P., Guadagnini, P.F. Annamostino, P. and Stonfer, M. (2004) : Severe E.colioiii septicemia and polyserositis in hens at the start lay. Avian pathology 29,311-317 


\section{الملخص العربي}

الأسباب البكتيريـة لانخفاض انتاج البيض في الدجاج البياض و الوقايـة بالتحصين في هذه الدراسة تم التعرف علي اهم الامراض البكتيرية و نأثيرها علي إنتاج البيض و الوقاية من الميكروب القولونى بالتحصين.

تم فحص 25 مزرعة دجاج بياض نعاني من نقص في انتاج البيض يتراوح من 3-10 \% و ارتفاع في نسبة الوفيات وقد تم عزل البكتربا كالآتي:

E.coli (21.9\%), Staphylococcus aureus (17.2\%),Pseudomonas aerogenosa (11.3\%), Proteus vulgaris (6.74\%) Enterococci $(5.61 \%)$ Klebsiellaoxytoca (5.41\%)Proteus mirabilis (5.41\%), Klebsellapnemoniae (5\%) Klebsiellaozaenae (5\%), Yersinia enterocolitica (4.18\%), Salmonella (4.08\%), Streptococcus species (3.77\%), Actinomycesbiogenes(3.06\%) and Citrobacterfreundii(1.22\%)

وقد صنفت معزولات الميكروب القولوني (215) كالآتي: $0166,0146,018,078,01,086$ and 020 وتم اختبار ضـراوة هذه المعزولات في كتاكيت عمر 9 ايام حيث كانت العترة (0166) هي الاكثر ضراوة و كانت نسبة الوفيات و الطيور المصابة ظاهريا عاليـة (42.9\%-42.9\%) مقارنـة بالعترات الاخري و تلاها في الخطورة العثرة (018) ثم (078) ثم (0146) ثم (020) ثم (01) و أخيرا العترة (086). وبالعدوي التجريبية في الدجاج البياض بالعترة 0166 كان هناك انخفاض معنوي في انتاج البيض بنسبة تتراوح بين (13.5\%-35\%) من الاسبوع الاول بعد العدوي الي نهايه التجربة ثم تلاهـا باقل خطورة علي الإتناج العترات 078 و 018 و 01 .كما اظهر التحصن بلقاح محضر معمليا منـالعترات (078 ، 0146 ، 01 ، 0166) كفاءة عاليـة ضد عدوي الميكروب القولوني حيث وجد ارتقاع معنوي في انتاج البيض بالقطيع المحصن والمعدي بالعترة 0166 كذللك ادي التحصين الي تقليل نسبة اعادة عزل الميكروب القولوني من الزرق و العزل من البيض. 